\title{
Prevalence and predictors of dyslipidemia on HAART and HAART naive HIV positive clients attending Debre Tabor Hospital, Debre Tabor, Ethiopia
}

\section{Anemut Tilahun Mulu ( $\nabla$ anemuttilahun@gmail.com )}

Debre Tabor University https://orcid.org/0000-0003-0496-6884

Getachew Yideg Yitbarek

Debre Tabor University

Fitalew Tadele Admasu

Debre Tabor University

Chalachew Yenew Denekew

Debre Tabor University

Biruk Demissie Melese

Debre Tabor University

\section{Research}

Keywords: Dyslipidemia, HAART, HIV / AIDS, Debre Tabor, Ethiopia

Posted Date: March 30th, 2021

DOI: https://doi.org/10.21203/rs.3.rs-344322/v1

License: (c) (i) This work is licensed under a Creative Commons Attribution 4.0 International License.

Read Full License 


\section{Abstract}

Background: HAART has been reported to be associated with a number of side effects in human immunodeficiency virus patients among which dyslipidemia is a common metabolic disorder.

Methods: A facility based comparative cross-sectional study among 228 HIV positive persons was conducted from July to August 2020. Socio-demographic and clinical data were collected using structured questionnaires. Fasting venous blood sample was drawn for Lipid profiles and CD4 cell determination. Anthropometric measurement was done. Data was analyzed using SPSS version 22 for windows. Result: A total of 228 HIV patients were enrolled in the study. Prevalence of dyslipidemia in HAART naive and on HAART HIV positive persons was 61(53.5\%) and 84 (73.7\%), respectively. The prevalence of TC $\geq 200 \mathrm{mg} / \mathrm{dl}$ was $50 \%$ and $30 \%$; HLD-c $<40 \mathrm{mg} / \mathrm{dl}$ was $43.8 \%$ and $36 \%$; LDL-c $\geq 130 \mathrm{mg} / \mathrm{dl}$ was $48.3 \%$ and $28.1 \%$; and TG $\geq 150 \mathrm{mg} / \mathrm{dl} 59.6 \%$ and $39 \%$ among on HAART and HAART naïve, respectively. Age greater than 40 years $(A O R=3.27,95 \%$ C.l: $1.47-7.25)$, blood pressure $\geq 140 / 90(A O R=$ 16.13, 95\% C.I: 5.81 - 44.75), being on HAART (AOR = 2.73, 95 \% C.I: 1.35 - 5.53) and body mass index > $25 \mathrm{~kg} / \mathrm{m}^{2}(\mathrm{AOR}=1.92,95 \%$ C.I: $1.20-4.81)$ were identified as determinants of dyslipidemia.

Conclusion: The mean value of lipid profile was significantly higher among HIV positive clients on HAART as compared to those HAART naïve HIV positive clients.

\section{Background}

Acquired Immune Deficiency Syndrome (AIDS) has become a global public health concern [1]. The different HAART regimens being used by many countries include a combination of at least three drugs such as protease inhibitors (PI), non-nucleoside reverse transcriptase inhibitors (NNRTI) and nucleoside reverse transcriptase inhibitors (NRTI)[1] . The introduction and extensive utilization of HAART in the treatment of HIV infection has led to significant reduction in AIDS-related morbidity and mortality[2].

Nowadays the World Health Organization (WHO) recommends the combination of two NRTI with a NNRTI [3]. Pls have become compulsory component of second-line treatment subsequent to failure of first-line regimens in which a change from Tenofovir/Lamivudine/Efavirenz (TDF/3TC/EFV) preferred first line combination to Tenofovir/Lamivudine/Lopinavir/r (TDF/3TC/LPV/r) second line combination [3]. Despite the pivotal role of HAART in the treatment of HIV infection, the use of HAART is reported to be associated with a series of side effects in HIV/AIDS infected clients [4]. The use of HAART is associated with abnormal changes in lipid profile in PLHIV [5]. Some of the lipid abnormalities include; elevated level of total cholesterol (TC), low-density lipoprotein-cholesterol (LDL-C), triglyceride (TG) and decreased level of high-density lipoprotein-cholesterol (HDL-c) [3]. Use of stavudine (d4T) and PIs have been reported to cause an elevation of the blood levels of TC, LDL-C, and TGs with variable effects on levels of HDL-C [6]. Use of Nevirapine has been associated with increased LDL-C, whereas use of long term use of efavirenz for long period of time has been associated with increase TC and TG[7]. 
Mechanism for the development of lipid abnormality is still unclear and proposed to be multi factorial[8]. The individual contributions of HIV infection, specific ARV agents, host genetics and changes in body composition all should be considered for the development of lipid abnormality among PLHIV [9]. These high levels of lipids increase the likelihood of developing blood clots, heart disease and heart attack, stroke and pancreatitis [1].

Most deaths among HIV positive clients are from liver disease, kidney disease and cardiovascular complications with factors related to the virus, the host and ART factors[10]. All HIV patients with dyslipidemia may not require lipid lowering therapy. The goal of lipid lowering therapy is to minimize an individual's cardiovascular risk. So, treatment of HIV-associated dyslipidemia should be a component of an attempt to improve cardiovascular health. Continuous monitoring and advice on diet and exercise, smoking cessation, management of hypertension and diabetes if present and use of anti-platelet agents are crucial measures in managing HIV associated dyslipidemia [11].

Although WHO ART guidelines do not include lipid monitoring in HIV treatment, lipid monitoring should be included in patients receiving HAART [4]. The prevalence of dyslipidemia on HAART individuals in resource-limited settings also has not been well characterized[12]. This study was aimed at identifying risk factors of dyslipidemia in HIV patients and determining whether HIV patients on HAART have high level of dyslipidemia compared to HAART naïve HIV positive clients [13].

\section{Methodology}

\subsection{Study Area and Period}

A facility based cross-sectional study was conducted in Debre Tabor Referral Hospital from July to August 2020.

\subsection{Sample Size and Study Subjects}

The sample size was determined using two population proportion formula by considering the following assumptions: proportion of HIV positive persons on HAART with LDL-c $\geq 130$ (40.8\%) [3] and proportion of HIV positive persons naive for HAART with LDL-c $\geq 130$ (21\%) [11]. Level of significance $(a / 2)=5 \%$; power $(1-\beta)=90 \% ; r=($ Ratio of exposed: none exposed $)=1: 1$. By adding $10 \%$ non-response rate the final sample size was 228 (114 on HAART and 114 HAART naïve HIV positive clients).

All HIV positive persons (age $\geq 18$ years) who were available during the study period were included. HIV patients with known DM, renal failure and on anti TB drugs were excluded from the study. HIV positive persons on HAART who change their regimen in less than a year and who took HAART for less than a year were excluded from the study.

\subsection{Data Collection Techniques and Instruments}


One BSc-nurse for socio-demographic data collection and two laboratory technologists for laboratory analysis were assigned throughout the data collection period. Structured questionnaire was used for socio-demographic data collection. Anthropometric measurements, height and weight were done.

\subsection{Blood Specimen Processing and Analysis}

A fasting venous blood sample was drawn for lipid profile and CD4 cell determination in separate test tube using vacutainer blood collection system. EDTA anti-coagulated venous blood was processed for CD4 count using BD FACS Count ${ }^{\mathrm{TM}}$ (BD USA). The TC/HDL-C ratio was also calculated. Lipid profile was assessed through enzymatic Methods using Clinical chemistry Analyzer (HumStar80, Canada).

According to the US National Cholesterol Education Program, Adult Treatment Panel III (NCEP-ATP III) guidelines, abnormal lipid profile was defined as TC $\geq 200 \mathrm{mg} / \mathrm{dl}, \mathrm{HDL}-\mathrm{c}<40 \mathrm{mg} / \mathrm{dl}, \mathrm{LDL}-\mathrm{C} \geq 130 \mathrm{mg} / \mathrm{dl}$, $\mathrm{TG} \geq 150 \mathrm{mg} / \mathrm{dl}, \mathrm{TC} / \mathrm{HDL}-\mathrm{c}$ ratio $\geq 5$ [8]. An elevation of any one of the lipid parameters above these limits was considered as dyslipidemia [7].

\subsection{Data Quality Assurance}

Questionnaire was translated to the local Amharic language and the Amharic version was used for the interview. Weight measurement was done using a digital scale to the nearest $0.1 \mathrm{~kg}$. A fixed base calibrated height scale was used for height measurement to the nearest $0.1 \mathrm{~cm}$. Measurements were done twice and the mean values were used to compute BMI. All reagents were checked for expiry date.

\subsection{Statistical Analysis}

Data was coded and entered using Epi-Info version 3.5.6 and then exported to SPSS version 22 for analysis. Descriptive statistics using frequency distribution was done for socio-demographic and clinical characteristics of study participants. Binary and multivariate logistic regression and independent student's T-test were done. A P value $<0.05$ were considered as statistically significant.

\section{Results}

\subsection{Socio-demographic and Characteristics of Study Participants}

A total of 228 HIV positive persons were enrolled in this study from whom, majority, 125 (54.82 \%) were females. Majority of study participants were within the age group of 31-40 years. The mean age of study participants was $35.31( \pm 7.20)$. The majority of patients can read and write.

Majority $(61.4 \%)$ of the patients are urban residents. 29 (12.8\%) of the patients had smoking history (Table 1). 
Table 1

Socio-demographic characteristics of HIV patients on HAART and HAART naïve in Debre Tabor referral hospital, Debre Tabor, Ethiopia, 2020.

\begin{tabular}{|c|c|c|c|}
\hline \\
\hline Variable & Categories & On HAART & HAART naïve \\
\hline \multirow[t]{2}{*}{ Sex } & Male & 53 & 50 \\
\hline & Female & 61 & 64 \\
\hline \multirow[t]{3}{*}{ Age } & $18-30$ & 24 & 32 \\
\hline & $31-40$ & 67 & 62 \\
\hline & $>40$ & 31 & 20 \\
\hline \multirow[t]{3}{*}{ Educational status } & Illiterate & 15 & 19 \\
\hline & Read and write & 69 & 70 \\
\hline & Diploma \&above & 30 & 25 \\
\hline \multirow[t]{2}{*}{ Residence } & Rural & 46 & 42 \\
\hline & Urban & 68 & 72 \\
\hline \multirow[t]{2}{*}{ Smoking habit } & Yes & 17 & 12 \\
\hline & No & 97 & 102 \\
\hline \multirow[t]{2}{*}{ Physical exercise } & Yes & 39 & 42 \\
\hline & No & 75 & 72 \\
\hline
\end{tabular}

\subsection{Clinical characteristics of study participants}

49 (21.5\%) of the study participants were obese. 99 (43.4\%) of the study participants were hypertensive. $68(29.8 \%)$ of the participants have a CD 4 count of $<350$ cells $/ \mathrm{mm}^{3}$, while $103(45.2 \%)$ have a CD 4 count of $350-550$ cells $/ \mathrm{mm}^{3}$ and 57 (25\%) of the participants have a CD4 count of $>550$ cells $/ \mathrm{mm}^{3}$ (Table 2). 
Table 2

clinical parameters of HIV patients on HAART and HAART naïve in Debre Tabor referral hospital, Debre Tabor, Ethiopia, 2020.

\begin{tabular}{|c|c|c|c|}
\hline Variable & Categories & On HAART & HAART naïve \\
\hline \multirow[t]{3}{*}{$\mathrm{BMI}\left(\mathrm{Kg} / \mathrm{m}^{2}\right)$} & $<18.5$ & 5 & 9 \\
\hline & $18.5-25$ & 78 & 87 \\
\hline & $>25$ & 31 & 18 \\
\hline \multirow[t]{2}{*}{ Blood pressure (MmHg) } & $<140 / 90$ & 61 & 68 \\
\hline & $\geq 140 / 90$ & 53 & 46 \\
\hline \multirow[t]{3}{*}{ CD4 cells $/ \mathrm{mm}^{3}$} & $<350$ & 19 & 49 \\
\hline & $350-550$ & 52 & 51 \\
\hline & $>550$ & 43 & 14 \\
\hline
\end{tabular}

\subsection{Lipid Profile of study participants}

Majority (63.6\%) of the study participants have dyslipidemia. 91(39.9\%) of the study participants have a total cholesterol of $\geq 200 \mathrm{mg} / \mathrm{dl}$. 91 (39.9\%) of study participants have diminished level of HDL-c (< 40 $\mathrm{mg} / \mathrm{dl}) .87(38.1 \%)$ of the study participants have elevated level of LDL-c (>130 mg/dl). $112(49.1 \%)$ of the study participants have elevated level of TG (>150 mg/dl) (Table 3$)$. 
Table 3

Serum lipid profile of study population by HAART status in Debre Tabor referral Hospital, Debre Tabor -Ethiopia from July 20 to August 13, 2020.

\begin{tabular}{|llll|}
\hline Variable & Categories & On HAART No (\%) & HAART naïve No (\%) \\
\hline Total dyslipidemia & Absent & $30(26.3)$ & $53(46.5)$ \\
\cline { 2 - 4 } & Present & $84(73.7)$ & $61(53.5)$ \\
\hline TC & $<200 \mathrm{mg} / \mathrm{dl}$ & $57(50)$ & $80(70.2)$ \\
\cline { 2 - 4 } & $\geq 200 \mathrm{mg} / \mathrm{dl}$ & $57(50)$ & $34(29.8)$ \\
HDL-C & $<40 \mathrm{mg} / \mathrm{dl}$ & $50(43.85)$ & $41(36)$ \\
& $\geq 40 \mathrm{mg} / \mathrm{dl}$ & $64(56.15)$ & $73(64)$ \\
LDL-c & $<130 \mathrm{mg} / \mathrm{dl}$ & $59(51.75)$ & $82(71.9)$ \\
& $\geq 130 \mathrm{mg} / \mathrm{dl}$ & $55(48.25)$ & $32(28.1)$ \\
TG & $<150 \mathrm{mg} / \mathrm{dl}$ & $46(40.4)$ & $70(61.4)$ \\
& $\geq 150 \mathrm{mg} / \mathrm{dl}$ & $68(59.6)$ & $44(38.6)$ \\
\hline
\end{tabular}

N.B: NO $=$ Number, HAART $=$ highly active antiretroviral therapy, TC $=$ total cholesterol, HDL-C $=$ HighDensity Lipoprotein-cholesterol, LDL-C = Low-Density Lipoprotein-cholesterol, TG = triglyceride, TC/HDL-c ratio $=$ total cholesterol/High-Density Lipoprotein-cholesterol ratio

\subsection{Associated Risk Factors of Dyslipidemia}

Among the study participants involved, 84 (73.68\%) on HAART and 61 (53.51\%) HAART naive persons had at least one lipid abnormality. The prevalence of dyslipidemia on HAART for TC, LDL-c \& TG was greater than HAART naïve which was significantly different. The Mean value of TC, LDL-C and TG were higher in persons on HAART than in HAART-naïve whereas the mean value of HDL-c was higher among HAART-naïve than on HAART HIV positive persons (Table 3 \&4).

Table 4. Comparison of the mean of variables by HAART status at Debre Tabor Hospital, Debre Tabor -Ethiopia from July 20 to August 13, 2020. 


\begin{tabular}{|c|c|c|c|c|}
\hline Variable & Group & Mean (SD) & $\mathrm{t}(95 \% \mathrm{CI})$ & P-value \\
\hline \multirow[t]{2}{*}{ Age in years } & HAART naïve & $34.42(6.49)$ & & \\
\hline & On HAART & $36.3(7.81)$ & $1.9(0.01,3.74)$ & 0.318 \\
\hline \multirow[t]{2}{*}{ TC } & HAART naïve & $186(24.09)$ & & \\
\hline & On HAART & $202(23.98)$ & $4.9(9.41,21.88)$ & $<0.001$ \\
\hline \multirow[t]{2}{*}{ HDL-C } & HAART naïve & $43.85(7.91)$ & & \\
\hline & On HAART & $40.52(6.09)$ & $-3.49(-5.18,-1.53)$ & $<0.001$ \\
\hline \multirow[t]{2}{*}{ LDL-C } & HAART naïve & 111.35 & & \\
\hline & On HAART & $126(22.86)$ & $4.6(8.84,19.81)$ & $<0.001$ \\
\hline \multirow[t]{2}{*}{ TG } & HAART naïve & $155(23.34)$ & & \\
\hline & On HAART & $175(36.81)$ & $5.11(11.90,28.16)$ & $<0.001$ \\
\hline \multirow[t]{2}{*}{ CD4 Cells/mm } & HAART naïve & $339(75.88)$ & & \\
\hline & On HAART & $500(157.25)$ & $9.9(128.55,193.13)$ & $<0.001$ \\
\hline \multirow[t]{2}{*}{$\mathrm{BMI}$ in $\mathrm{kg} / \mathrm{m} 2$} & HAART naïve & $22.11(2.63)$ & & \\
\hline & On HAART & $23.33(2.72)$ & $3.4(0.54,1.92)$ & 0.862 \\
\hline
\end{tabular}

Multivariable logistic regression analysis was done for variables having $p<0.25$ in binary logistic regression analysis (Age, CD4 count, BMI, blood pressure, and HAART status). After controlling potential confounding factors in multiple logistic regression analysis; age greater than 40 years old (AOR $=3.27$, 95\% C.I: $1.47-7.25)$, blood pressure $\geq 140 / 90$ (AOR $=16.13,95 \%$ C.I: $5.81-44.75)$, being on HAART (AOR $=2.73,95 \%$ C.I: $1.35-5.53)$ and body mass index $>25 \mathrm{~kg} / \mathrm{m} 2$ ( $\mathrm{AOR}=1.92,95 \%$ C.l: $1.20-4.81)$ were identified as determinants of Dyslipidemia (Table 5).

Table 5. Associations of variables with dyslipidemia among persons on HAART and HAART naive in Debre Tabor Hospital, Debre Tabor -Ethiopia from July 20 to August 13, 2020

Outcome variables in $\mathrm{mg} / \mathrm{dl}$ (95 \% CI) 
Explanatory

Variable

\section{TC $\geq 200 \quad$ HDL-c $<40 \quad$ LDL-c $\geq 130 \quad$ TG $\geq 150$}

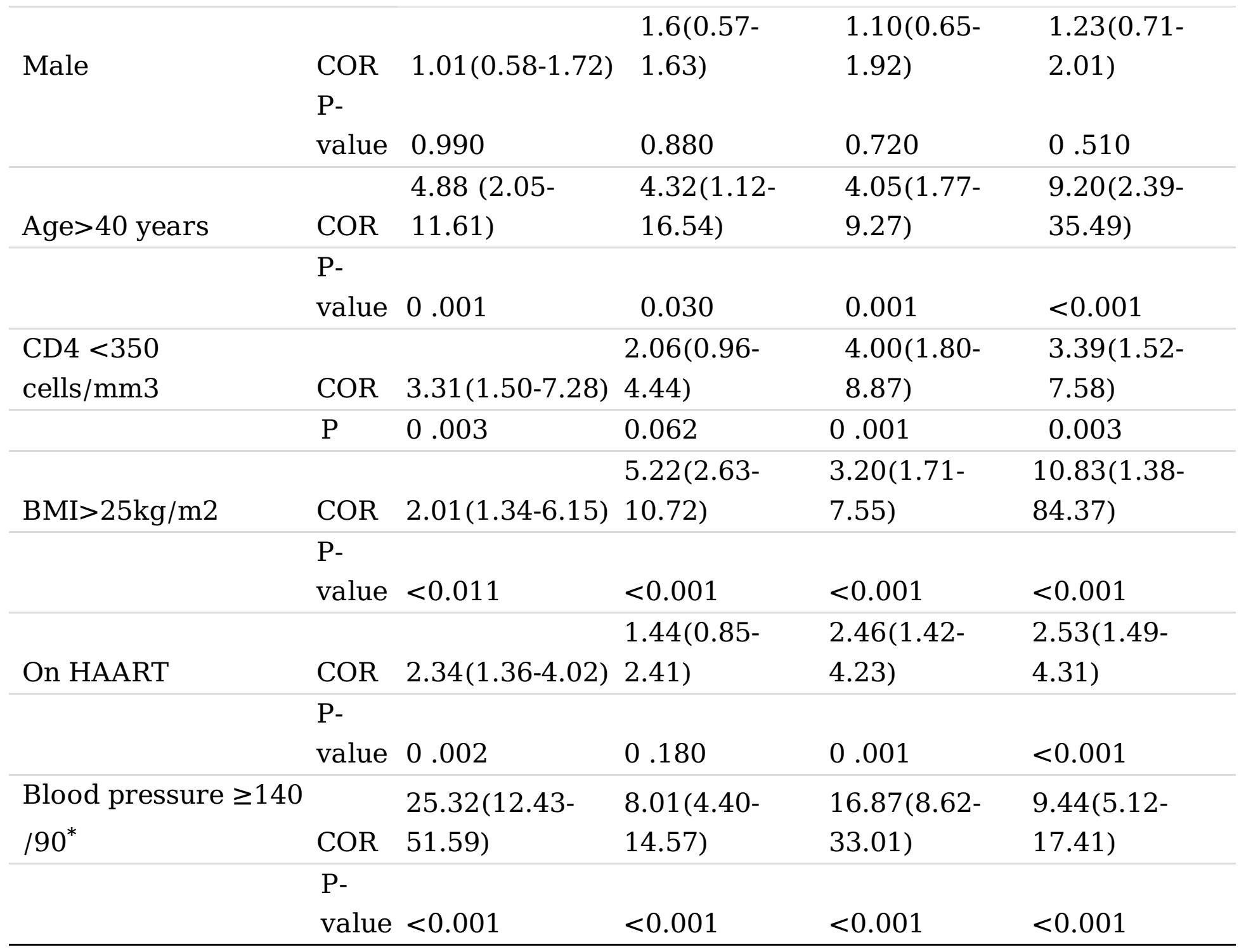

N.B: $\mathrm{COR}=$ Crude Odes Ratio, $\mathrm{CI}=$ Confidence Interval, $\mathrm{TC}=$ total cholesterol, HDL-c $=$ High-Density Lipoprotein-cholesterol, LDL$\mathrm{C}=$ Low-Density Lipoprotein-cholesterol, TG = triglyceride, BMI = Body Mass Index, HAART = highly active antiretroviral therapy,

*blood pressure as systolic/diastolic

Table 6. Determinant factors of Dyslipidemia and lipid profile level among HIV positive persons, adjusted for the potential confounding factors of study participants in Debre Tabor Hospital, Debre Tabor -Ethiopia from July 20 to August 13, 2020 


\section{Explanatory \\ Variable}

lipid profile level in $\mathrm{mg} / \mathrm{dl}$

(95\% CI)

Dyslipidemia

\section{HDL-c $<$ LDL-c $\geq$}

$40 \quad 130$
$\mathrm{TG} \geq 150$

$2.44(1.06-$

$5.64)$

Age $>40$ years old AOR 7.25)

$3.27(1.47-$

P-

value 0.004

0.037

\begin{tabular}{|c|c|c|c|c|c|c|}
\hline & value & & & & & \multirow{3}{*}{$10.83(1.38$} \\
\hline \multirow[b]{2}{*}{$\mathrm{BMI}>25 \mathrm{~kg} / \mathrm{m} 2$} & \multirow[b]{2}{*}{ AOR } & \multirow{2}{*}{$\begin{array}{l}1.92(1.20- \\
4.81)\end{array}$} & \multirow{2}{*}{$\begin{array}{l}2.31(1.04- \\
7.66)\end{array}$} & & \multirow{2}{*}{$\begin{array}{l}3.20(1.71- \\
7.55)\end{array}$} & \\
\hline & & & & & & \\
\hline & & & & & & $84.37)$ \\
\hline & $\mathrm{P}-$ & & & & & \\
\hline & value & 0.044 & 0.025 & & $<0.001$ & $<0.001$ \\
\hline \multirow{4}{*}{ On HAART } & & 2.73(1.35- & $3.15(1.49-$ & & $3.02(1.42-$ & 2.87(1.51- \\
\hline & AOR & 5.53) & 6.68) & & $4.23)$ & $5.45)$ \\
\hline & $\mathrm{P}-$ & & & & & \\
\hline & value & 0.005 & 0.003 & & 0.002 & 0.001 \\
\hline \multirow{4}{*}{$\begin{array}{l}\text { Blood pressure } \\
\geq 140 / 90\end{array}$} & & 16.13(5.81- & 28.59(13.39- & $5.24(2.69-$ & 18.44(9.10- & 7.08(3.45- \\
\hline & AOR & 44.75) & & & & 14.53) \\
\hline & & & $61.04)$ & 10.22) & $37.41)$ & \\
\hline & $\begin{array}{l}\mathrm{P}- \\
\text { value }\end{array}$ & $<0.001$ & $<0.001$ & $<0.001$ & $<0.001$ & $<0.001$ \\
\hline
\end{tabular}

N.B: AOR = Adjusted Odes Ratio, CI = Confidence Interval, TC = total cholesterol, HDL-c = High-Density Lipoprotein-cholesterol, LDL-c = Low-Density Lipoprotein

\section{Discussion}

The prevalence of dyslipidemia in this study was 145 (63.60\%), of them 84 (73.68\%) were on HAART and 61 (53.51\%) were HAART naïve HIV positive clients. The mean value of TC, LDL-C and TG were higher in HIV clients on HAART than those who are not on HAART. The mean value of HDL-c was higher among HAART-naïve HIV clients than those on HAART. In this study the mean value of CD4 cell count on HAART group was significantly different from the HAART naïve HIV positive clients. But, CD 4 cell count was not significantly associated with dyslipidemia and lipid profile level. This might be due to HAART effect on improving immunological status of HIV clients [14].

In this study the mean value of each lipid profile was significantly higher among HIV clients on HAART than HAART naïve HIV clients which was similar with a study conducted in Sao Paulo, Brazil[15]. In this study we found that the proportions of raised TC, LDL-C, TG and TC/HDL-C ratio were significantly higher among HIV clients on HAART when compared to HAART naive HIV clients which indicates the atherogenic effect of HAART for the development of cardiovascular diseases[16]. The association between HAART 
and adverse lipid profile has been largely described for regimens when hypothetically switching patients from first-line to second-line treatment [17].

HIV patients on HAART with TC $\geq 200 \mathrm{mg} / \mathrm{dl}(50 \%)$ was higher than the findings reported from; Cameron (37.7\%), Hawassa-Ethiopia (43.4\%) \& Jimma-Ethiopia (6.4\%) [3]. This variation might be due to the sampling technique and the cut of value used. A prospective study conducted in India for the effects of NNRTIs indicated that the proportion of patients with TC $\geq 200 \mathrm{mg} / \mathrm{dl}$ had increased from $1-26 \%$ after 12 month of treatment [18].

The prevalence of HDL-c $<40 \mathrm{mg} / \mathrm{dl}$ among HIV clients on HAART was $43.8 \%$. This is comparable with the prevalence reported from Hawassa-Ethiopia which is 43.4\% [3]. However, the prevalence of HDL-c< $40 \mathrm{mg} / \mathrm{dl}$ among HIV clients on HAART is higher than the prevalence reported from Jimma-Ethiopia (32.6\%)[11]. The prevalence of LDL-c $\geq 150 \mathrm{mg} / \mathrm{dl}$ in our HAART group was $48.3 \%$ which is comparable with the prevalence reported from Cameron (46.4\%) [17]. The prevalence of raised TG on HAART group was $59.6 \%$, which is comparable with the report from Hawassa-Ethiopia (55.8\%) But was higher than studies done in; India, Cameron, Jimma-Ethiopia reported 32\%, 39\%, 18.2\%, respectively[3, 11]. This might be due to difference in magnitude of lipid profile derangements induced by HAART with duration of treatment, across populations and setting.

\section{Conclusion}

The raised levels of TC, LDL-C and TG values were significantly associated with utilization of HAART regimens. The mean value of all lipid parameters was significantly higher among HIV clients using HAART when compared to those HIV clients who do not use HAART regimens. Old age, raised BP, being on HAART and increased in BMI were determinant factors of dyslipidemia among HIV clients. So, further studies with long term follow-up are vital to explain more on the causes of dyslipidemia and the pattern of lipid profile changes with HAART in HIV clients in resource limited countries.

\section{Abbreviations}

ARV- Anti-Retro-Viral

CART- Combined anti-retroviral therapy

HAART- Highly Active Anti-Retroviral Therapy

HDL- High Density Lipoprotein

HIV- Human Immune-Deficiency Virus

LDL- Low Density Lipoprotein

NNRTI- Non-Nucleoside Reverse Transcriptase Inhibitors 
NRTI- Nucleoside Reverse Transcriptase Inhibitors

PI- Protease Inhibitor

TC- Total Cholesterol

TG- Triglycerides

WHO- World Health Organization

\section{Declarations}

\section{Data Availability}

The SPSS data used to support the findings of this study are available from the corresponding author upon request.

\section{Consent to publish}

Not applicable

\section{Ethical approval}

Ethical approval was obtained from Debre Tabor University Ethical Review Board and a letter of permission to conduct the study was obtained from Debre Tabor Hospital clinical director office. Written informed consent was obtained from each study participants

\section{Conflict of interest}

The authors declare that they have no competing interests.

\section{Funding}

All the funds required to conduct this study was fulfilled by Debre Tabor University.

\section{Author's contributions}

Anemut- perform data entry, analysis and interpretation.

Getachew- perform the statistical analysis and data interpretation in collaboration with Anemut.

Fitalew- accomplishes the manuscript write up.

Chalachew- participates in write up

\section{Acknowledgments}


Our deepest gratitude goes to data collectors for their invaluable effort. We would also like to acknowledge study subjects who were volunteer and took their time to give us all the relevant information for the study.

\section{References}

1. Melzi S, Carenzi L, Cossu MV, Passerini S, Capetti A, Rizzardini G, “Lipid Metabolism and Cardiovascular Risk in HIV-1 Infection and HAART: Present and Future Problems," vol. 2010, 2010.

2. Ji S, et al., "Changes in Lipid Indices in HIV + Cases on HAART," vol. 2019, 2019.

3. Tadewos A, Addis Z, Ambachew H, Banerjee S. "Prevalence of dyslipidemia among HIV-infected patients using first-line highly active antiretroviral therapy in Southern Ethiopia: a cross-sectional comparative group study," pp. 1-8, 2012.

4. Amberbir A, et al., "Dyslipidemia among rural and urban HIV patients in south-east Malawi," pp. 1-11, 2018.

5. Trevillyan JM, et al., "Changes in plasma lipidome following initiation of antiretroviral therapy," pp. 1-16, 2018.

6. Cahn P, et al., "ORIGINAL Metabolic profile and cardiovascular risk factors among Latin American HIV-infected patients receiving HAART," vol. 14, no. 2, pp. 158-166.

7. Zhang $M$, et al. Prevalence of dyslipidemia and achievement of low-density lipoprotein cholesterol targets in Chinese adults: A nationally representative survey of 163, 641 adults. Int J Cardiol. 2018;260:196-203.

8. Dave JA, Levitt NS, Ross IL, Lacerda M, Maartens G, Blom D. "Anti-Retroviral Therapy Increases the Prevalence of Dyslipidemia in South African HIV-Infected Patients," pp. 1-13, 2016.

9. Dube MP, et al., "Guidelines for the Evaluation and Management of Dyslipidemia in Human Immunodeficiency Virus (HIV) - Infected Adults Receiving Antiretroviral Therapy: Recommendations of the HIV Medicine Association of the Infectious Disease Society of America and the Adult AIDS Clinical Trials Group," vol. 37, pp. 613-627, 2003.

10. Bekolo CE, Nguena MB, Ewane L, Bekoule PS, Kollo B. "The lipid profile of HIV-infected patients receiving antiretroviral therapy in a rural Cameroonian population," 2014.

11. Mohammed IY, Yahaya IA. "Therapy - related lipid profile changes among patients ' on highly active antiretroviral treatment in Kano, North - Western Nigeria," pp. 111-115, 2015.

12. Thie R, Chu U, B. F-, and Re C. "Prevalence and factors associated with renal impairment in HIVinfected patients, ANRS C03 Aquitaine Cohort, France," pp. 308-317, 2010.

13. Access O. "Dyslipidemia in people living with HIV-AIDS in a tertiary hospital in South-East Nigeria," vol. 8688, pp. 1-7, 2017.

14. Shiferaw Y, et al., "Assessment of knowledge, attitude and risk behaviors towards HIV / AIDS and other sexual transmitted infection among preparatory students of Gondar town, north west Ethiopia," pp. 1-8, 2011. 
15. Iffen TS, Efobi H, Udonwa NE. "Lipid Profile of HIV-Positive Patients Attending University of Calabar Teaching Hospital, Calabar - Nigeria Department of Family Medicine, College of Medical Sciences," vol. 5, no. 4, pp. 89-93, 2010.

16. Riddler SA, et al., "Longitudinal changes in serum lipids among HIV-infected men on highly active antiretroviral therapy," pp. 280-287, 2007.

17. Nsagha DS, et al., "Highly active antiretroviral therapy and dyslipidemia in people living with HIV / AIDS in Fako Division, South West Region of Cameroon," BMC Cardiovasc. Disord., pp. 1-8, 2015.

18. Indumati V, Vijay V, Shekhanawar MS, Amareshwaras M, Shantala D. "Comparison of Serum Lipid Profile in HIV Positive Patients on ART with ART Naïve Patients," vol. 8, no. 10, pp. 8-11, 2014. 